\title{
Low noise SQUID simulator with large dynamic range of up to eight flux quanta
}

\author{
A. Martinez*, J. Flokstra, C. Rillo**, L.A. Angurel**, L.M. Garcia** \\ and H.J.M. ter Brake \\ Twente University of Technology, Department of Applied Physics, PO Box 217, 7500 AE \\ Enschede, The Netherlands \\ **ICMA and Facultad e Ciencias, University of Zaragoza, 50009 Zaragoza, Spain
}

\section{Received 17 July 1989; revised 3 November 1989}

\begin{abstract}
A 19 channel d.c. SQUID magnetometer for biomagnetic research is under construction. The system needs compactly built control and detection electronics and to facilitate the test an electronic circuit simulating the typical periodic characteristics of a SQUID was developed. In total nine steps of triangular shape were implemented with a step voltage reference generator and precision full-wave rectifier. The transfer function of the SQUID electronics and the maximum slew rate of the system could be easily measured.
\end{abstract}

Keywords: SQUIDs; instrumentation; SQUID electronics

Multichannel SQUID magnetometry requires compactly built preamplifiers and control and detection electronics. The authors are constructing a 19 channel d.c. SQUID magnetometer for brain research, and various schemes for the electronic processing have been designed. To facilitate a functional test and adjustment of the modules of the SQUID electronics, a SQUID simulator with a dynamic range of up to eight flux quanta has been designed. Several interesting advantages have been obtained. There is no need for a cryogenic infrastructure and there is no risk of destroying the sensor. Furthermore, the electronic system can be simply extended to a multichannel version so that 19 SQUIDs with equal quality factors can be simulated. Typical transfer problems in multichannel systems such as cross-talk between transmission lines can be easily studied, It is clear that specific properties due to the SQUID configuration, such as resonances caused by the coupling between the SQUID loop and planar coupling coil, cannot be simulated. Also, the $1 / f$ noise cannot be considered. Despite these two shortcomings, the SQUID simulator is a powerful instrument in multichannel SQUID instrumentation.

In several papers ${ }^{1,2}$ electronic circuits have been described that represent the functional behaviour of Josephson junctions and SQUIDs. The various terms in the non-linear differential equations are simulated by electronic networks, making it possible to study the effect of all relevant parameters. This leads to rather complicated designs. For testing the SQUID read-out electronics such an overall approach is not necessary because only the flux-voltage transfer has to be simulated and much simpler circuits are adequate.

In this paper an electronic circuit is described which

*On leave from ETSI Industriales, University of Zaragoza, 50015 Zaragoza, Spain implements nine steps of the typical periodic characteristic of a SQUID. Although the implementation involves the triangular flux-voltage response of a RF SQUID system, there is no reduction in validity when d.c. SQUID electronics are tested with the simulator.

The use of a one-step SQUID simulator has been reported by Rillo et $a l^{3}$. The transfer function of the SQUID is replaced by a V shape voltage-flux curve, simulated with a precision full-wave rectifier (PFWR). However, the real transfer function is periodic with respect to the flux and the use of a multiplexed series of PFWRs was suggested to obtain this behaviour.

In the present design only one PFWR is used for the generation of all steps, leading to a reduction in the necessary components. Furthermore, the forward transfer ratio does not change from step to step. The nine periods were realized with a reference voltage step generator.

The forward transfer ratio, $S$, coupling between modulation coil and SQUID, $M_{\mathrm{f}}$, and dynamic resistance of the SQUID, $R_{\mathrm{d}}$, are all relevant quantities in a d.c. SQUID feedback loop operation. Typical values are: $S=100 \mu \mathrm{V} / \Phi_{\mathrm{o}}, M_{\mathrm{f}}=100.000 \Phi_{\mathrm{o}} / \mathrm{A}$ and $R_{\mathrm{d}}=4 \Omega$. These values have been implemented in the simulator.

The multi-step simulator enables the study of the maximum slew rate of the system. An input signal of known frequency and increasing amplitude is used to provoke the unlock. This will result in a monotonous increment of the flux error signal up to $\Phi_{0} / 4$, corresponding to the maximum limit for the locked operation. This situation is internally detected by the simulator and appropriately signalled with a LED lamp. By exceeding the slew rate limit the system unlocks and a new lock at another step may be obtained.

The content of this paper is as follows. First, the different parts of the circuit are described and it is shown how the main parameters $S, M_{\mathrm{f}}$ and $R_{\mathrm{d}}$ have been realized. Second, 
several tests of the simulator are reported. Then, in the last part of the paper, some conclusions will be drawn.

\section{Circuit description}

A schematic diagram of a d.c. SQUID configuration is presented in Figure 1, showing the input and feedback coils and the main electrical parameters involved. The input coil drives a current, $I_{i}$, and is coupled to the SQUID by $M_{\mathrm{i}}$. The corresponding quantities for the feedback coil are $I_{\mathrm{f}}$ and $M_{\mathrm{f}}$, respectively. $I_{\mathrm{b}}$ represents the bias current and $V_{0}$ the output voltage of the sensor.

An equivalent block scheme of the d.c. SQUID and the typical transfer function are given in Figures $2 a$ and $b$, respectively. The fluxes induced by the input and feedback currents are $\Phi_{i}(s=j \omega)$ and $\Phi_{\mathrm{r}}(s)$, respectively. $\Phi_{e}(s)$ is the resulting coupled flux. The voltage-flux characteristic is obtained in an open loop.

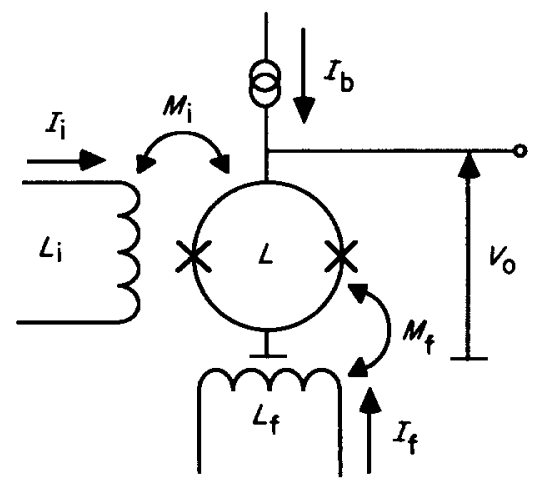

Figure 1 D.c. SQUID consisting of two Josephson junctions in a superconducting loop of inductance $L$. Currents $t_{i}$ and $/ f$ through input coil and feedback coil, respectively, couple magnetic flux into SQUID via mutual inductances $M_{\mathrm{i}}$ and $M_{\mathrm{f}}$, respectively. $I_{\mathrm{b}}$ is the bias current and $V_{0}$ the output voltage modulated by the flux

a

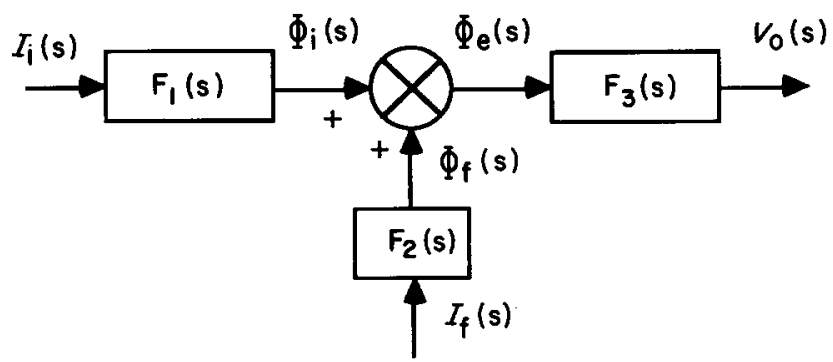

b

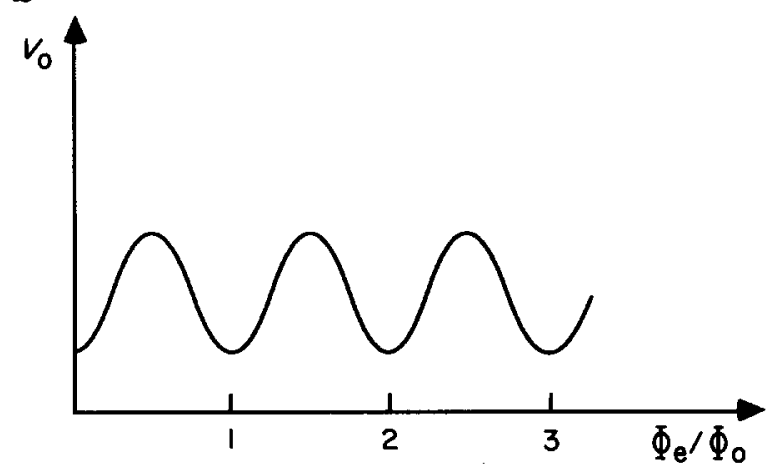

Figure 2 (a) Equivalent block scheme of the d.c. SQUID in Figure 1. $F_{1}(s=j \omega), F_{2}(s)$ and $F_{3}(s)$ are transfer functions. (b) $V_{0}$ versus $\Phi_{e} / \Phi_{0}$ for constant bias current. $\Phi_{0}$ is flux quantum a
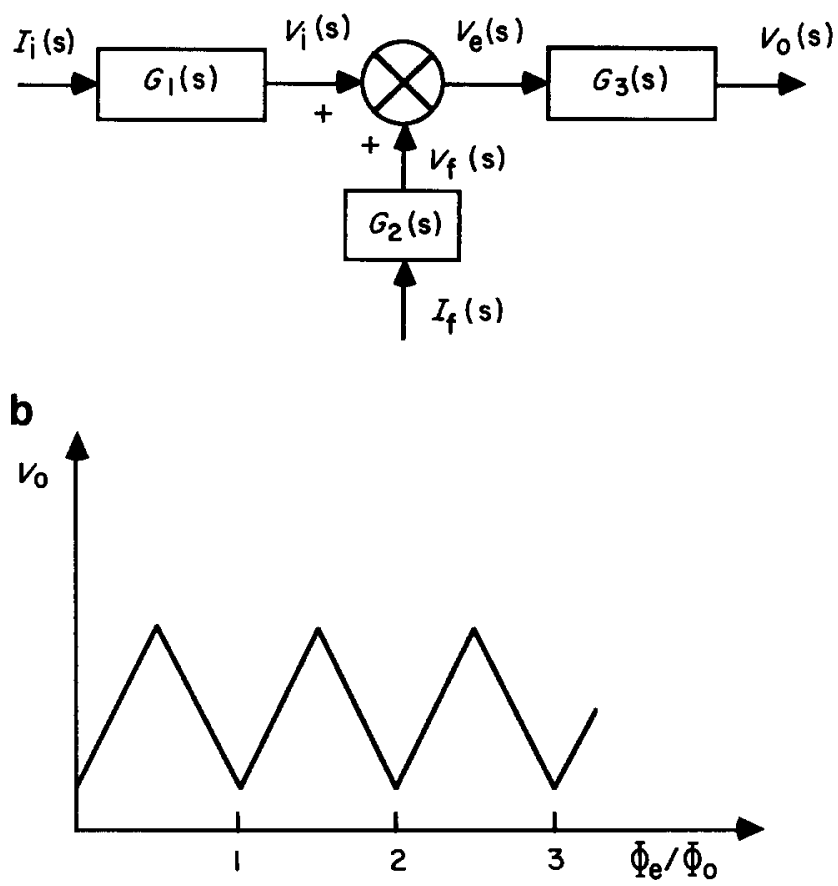

Figure 3 (a) Scheme equivalent to that of Figure $2 a$ with an internal representation of flux as a voltage. (b) Approximation of sinusoidal shape of Figure $2 b$ with a $V$ shape pattern

The inputs of the simulator are currents and the output is a voltage (Figure $3 a$ ) as holds for the d.c. SQUID. The transfer function of the d.c. SQUID is approximated by the triangular pattern of Figure $3 b$, which can be easily realized without disrupting the operating principle. Using the appropriate values for $M_{\mathrm{f}}, S$ and $R_{\mathrm{d}}$ in the design, as mentioned previously, the simulator can be directly coupled to the d.c. SQUID control electronics without any other interface.

As is seen from Figures $2 a$ and $3 a$, the flux coupled to the SQUID is represented by a voltage. It would be necessary to use two current-to-voltage converters and one voltage adder in the simulator to obtain the error signal, $V_{\mathrm{e}}(s)$. However, if $M_{\mathrm{i}}$ is taken to be equal to $M_{\mathrm{f}}$ (which can be done without loss of validity), only one current-to-voltage converter is necessary, with the adding point realized at its input. This simplification is used in the following considerations.

A schematic block diagram of the simulator, consisting of five blocks, is shown in Figure 4. Block (1) is a standard current-to-voltage converter built with an operational amplifier (OP amp) and a feedback resistor. The current, $I_{\mathrm{p}}$, appears in addition to $I_{\mathrm{i}}$ and $I_{\mathrm{f}}$ and is used as preset current. The preset current determines one particular point of the transfer function when no other signals are present at the input. The order of magnitude of the currents follows from the coupling factor $M_{\mathrm{f}}=10^{5} \Phi_{\mathrm{o}} / \mathrm{A}$; thus, $1 \Phi_{\mathrm{o}}$ is generated by a current of $10 \mu \mathrm{A}$. All currents are added at the negative input of the OP amp. Due to intrinsic limits of the components used, the uncertainty in offset voltages will be of the order of $3 \mathrm{mV}$, and in order to obtain an accuracy within $1 \%$ in the internal voltage repesentation of the flux quantum, $\Phi_{\mathrm{o}}$, a minimum transfer of $300 \mathrm{mV} / \Phi_{o}$ is necessary. Here, a transfer of $330 \mathrm{mV} / \Phi_{0}$ was chosen, leading to a feedback resistor of $33 \mathrm{k} \Omega$. 


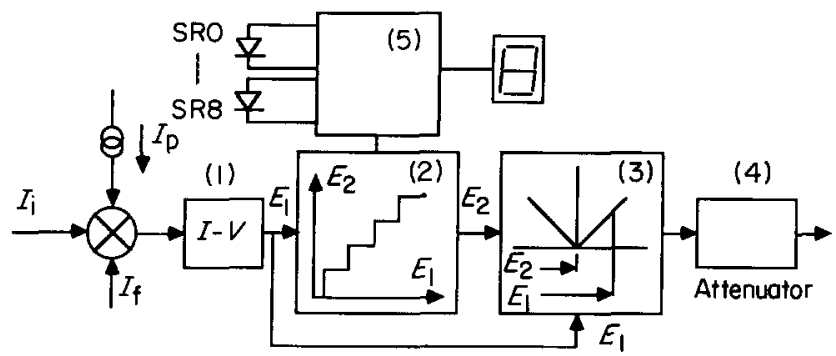

Figure 4 Modules of the simulator: (1), current-to-voltage converter; (2) generates discrete values of voltage references $E_{2}$; (3) absolute value circuit with differential input; (4) attenuator to adapt internal and external levels of signal; (5) logic circuit to display step in operation and slew rate occurrence

A low noise type (OP27) OP amp was selected which enabled work to be carried out with a modulation frequency of $100 \mathrm{kHz}$ with a square wave. A small compensation capacitor, empirically selected, should be placed in parallel with the feedback resistor to obtain an adequately shaped wave form.

Block (2) is a generator of voltage reference $E_{2}$, having discrete values of the form $E_{2}=n \times 330 \mathrm{mV}$, thus representing flux values of $n \Phi_{\mathrm{o}}$. Implementation involves a nine step simulator, so that $n=0,1, \ldots 8$. The value of $E_{2}$ holds for the interval $E_{1}=(n \times 330 \pm 165) \mathrm{mV}$ and thus for the flux interval $n \Phi \pm\left(n \Phi_{0} / 2\right)$. The actual value of $n$ is the step in which the simulator is working.

The layout of the step voltage generator and the output characteristic are given in Figures $5 a$ and $b$. An array of resistors of equal value, $R$, fed by a current source of value $I$, provides the series of reference voltages equivalent to $8 \Phi_{0}, 7.5 \Phi_{0}, 7 \Phi_{0}, \ldots 0.5 \Phi_{0}, 0$. The analog switches, SW1 and $\mathrm{SW} 2$, controlled by the voltage comparators, $\mathrm{VC}_{\mathrm{i}}$, select one of the integer values. The swapping occurs when $E_{1}$ exceeds a value equivalent to $(n+1 / 2) \Phi_{0}$. The accuracy of the representation of one $\Phi_{o}$ is determined by: 1 , the precision of the resistors, $R ; 2$, the ratio $V_{\text {off }} / R I$, where $V_{\text {off }}$ is the offset voltage of one voltage comparator; and $3,\left(\Sigma i_{b}\right) / I$, where $i_{\mathrm{b}}$ is the bias current of one voltage comparator.

Each bias current flows from the current source to the voltage comparator across the resistors, $R$. Consequently, because of $i_{\mathrm{b}}$ there is a mismatch in the voltage drop between two consecutive resistors of $R i_{\mathrm{b}}$ (maximum). For the two resistors at both ends of the array this difference will be $\Sigma i_{b} R$ in the worst case. Assuming an accuracy of $1 \%$ for $\Phi_{0}$, the resistors should at least have a precision of $1 \%$. Furthermore, point 3 will determine $I$, and points 2 and 3 will determine $R$. Taking typical values from the CPM-02 voltage comparator, the following values result: $R=2.2 \Omega, I=75 \mathrm{~mA}$.

Block (3) is an absolute value circuit (AVC) with differential input. The output of this block is given by $k\left|E_{1}-E_{2}\right|, k$ being a constant. If one of the inputs is 0 , the behaviour of this block is that of a full-wave rectifier. With $E_{1}$ from block (1) and $E_{2}$ from block (2), the nine steps are generated.

Intrinsic limitations of the rate of change between two consecutive levels of $E_{2}$ and delays due to the change of state of the AVC restrict the speed of change of $E_{1}$ to values of $\approx 1800$ steps per second. In cases of higher speed, the $\mathrm{V}$ shape pattern becomes disconnected. However, when the simulator operates in a closed loop

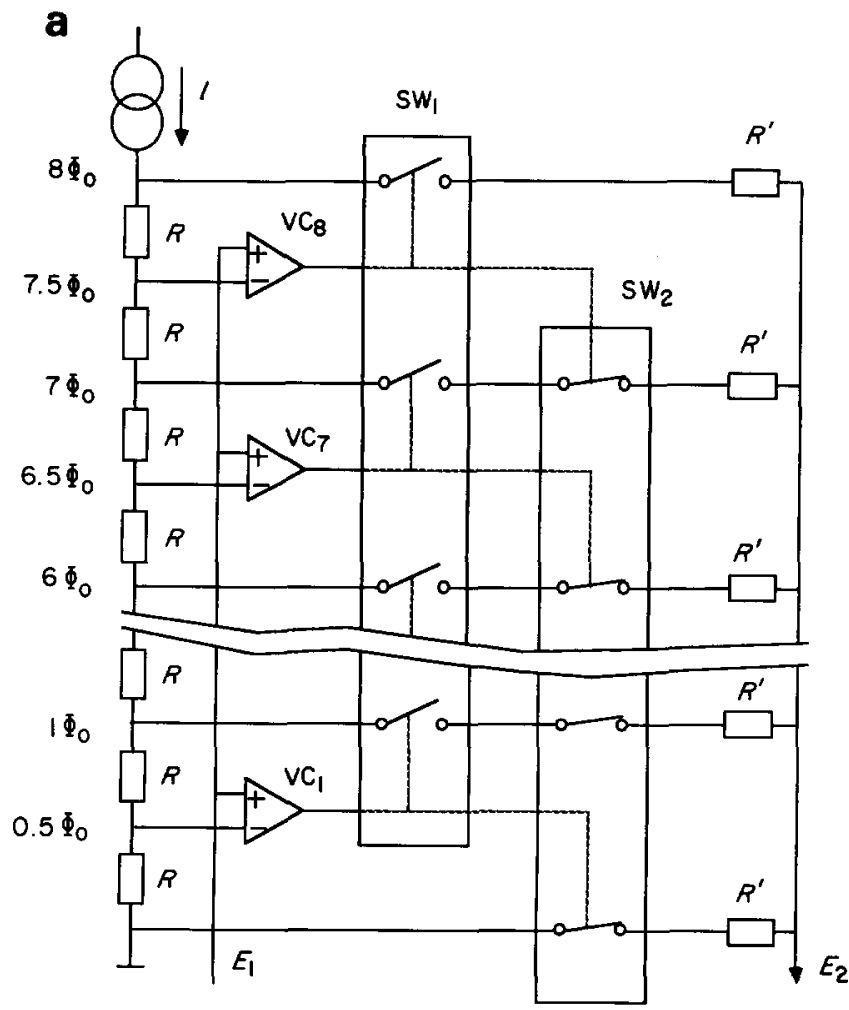

b

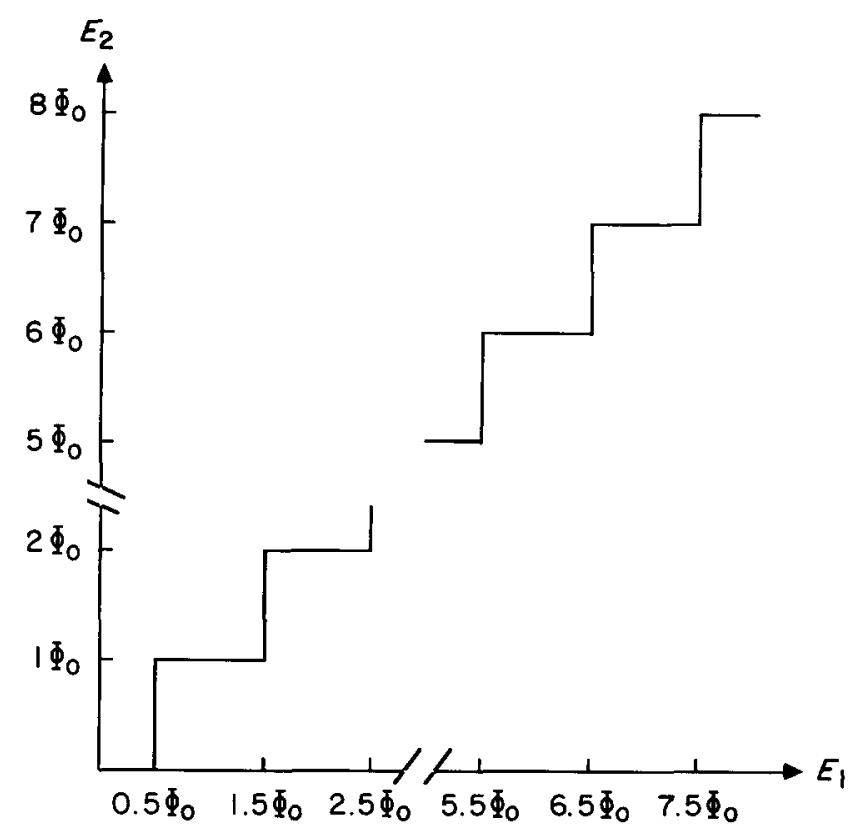

Figure 5 (a) Circuit that generates discrete voltage references $E_{2}$. Output voltage $E_{2}$ is determined by the activated voltage comparator with the highest number in the figure. (b) Characteristic obtained from the circuit in Figure 5a

only one step is involved and the limitations concerning $E_{2}$ are not relevant. There are several schemes for PFWRs. The best performance is obtained with the power supply current sensing technique, where the sensing is realized with a current mirror ${ }^{4,5}$. In this case the frequency behaviour is optimal and the distortion for low level signals is small.

In Figure 6 the scheme for the AVC is presented. Two matched low noise OP amps (OP1 and OP2 are OP 227 type) are used for obtaining the characteristics of a PFWR, 


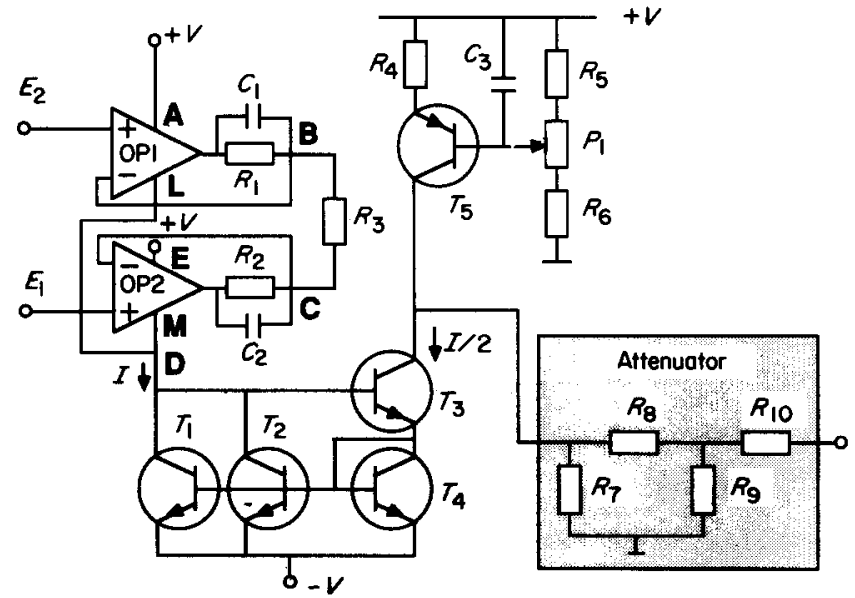

Figure 6 Scheme of absolute value circuit. Current sink, $I=\mid E_{2}-$ $E_{1} \mid / R_{3}$. It is sensed by the current mirror arrangement of T1, T2, T3 and T4 with a current ratio 0.5 . If $E_{2}=E_{1}$, current at collector of T3 is compensated with current source supplied by T5. Attenuator adapts the levels of internal and external signals. Current through $R_{\mathrm{g}}$ defines $S=100 \mu \mathrm{V} / \bar{\Phi}_{\mathrm{o}} . \boldsymbol{R}_{\mathbf{9}}+\boldsymbol{R}_{10}$ determines $\boldsymbol{R}_{\mathrm{d}}=\mathbf{4 \Omega}$. Attenuator has a load of $\approx 1 \Omega$ for the AVC

being in principle present in the current $I$. This current is sensed by the current mirror arrangement of the transistors $\mathrm{T} 1, \mathrm{~T} 2, \mathrm{~T} 3$ and $\mathrm{T} 4$, leading to the mirror current of $I / 2$. The accuracy for the current transfer ratio with four matched transistors is within $1 \%{ }^{6,7}$.

When $E_{1}=E_{2}$, there may still be a small current, $I$, due to the bias of the internal circuits of the two OP amps. However, the effect of this current is compensated with the current source provided by T5. The case $E_{1}<E_{2}$ results in a current flow through the path $A-B-C-M-D$, whereas $E_{1}>E_{2}$ leads to the path E-C-B-L-D. In both cases, the current at the output is given by $0.5\left|E_{1}-E_{2}\right| / R_{3}$. The value for $R_{3}$ is taken as $16.5 \Omega$ in order to obtain a sensed current at the input of the current mirror of $20 \mathrm{~mA} / \Phi_{0}$. This value is adequate for obtaining an almost non-rounded $\mathrm{V}$ shape.

Block (4) is an attenuator (Figure 6). It determines the two important parameters, the forward transfer ratio, $S$, and the dynamic resistance, $R_{\mathrm{d}}$. It is in fact a network of resistors which divides the current by a factor of 100 $\left(R_{7}=1 \Omega, R_{8}=98 \Omega, R_{9}=1 \Omega\right)$. The voltage drop at $R_{9}$ is $100 \mu \mathrm{V} / \Phi_{0}$, being the assumed forward transfer ratio. The output resistance is determined by $R_{9}+R_{10}$, and to obtain $R_{\mathrm{d}}=4 \Omega$ the value of $R_{10}$ has to be taken as equal to $3 \Omega$. The attenuator is a very small load for the AVC, producing a maximum voltage drop of $5 \mathrm{mV}$. This small swing of voltage results in ideal current source behaviour of T3 and T5, with only small distortion.

Block (5) is a circuit to display the step number in which the system is actually operating. It is also used to measure the value of the slew rate of the input signal necessary to unlock the system when it is working in a closed loop. The steps are identified from the discrete levels of $E_{2}$, and thus from the state of the voltage comparators. To signal the step number properly, the state of the eight comparators is codified into a seven segment display. This is realized with a nine to four priority encoder and a BCD to seven segment code converter (Figure 7).

To measure the slew rate, the simulator is connected to the control electronics in a closed loop. The $100 \mathrm{kHz}$

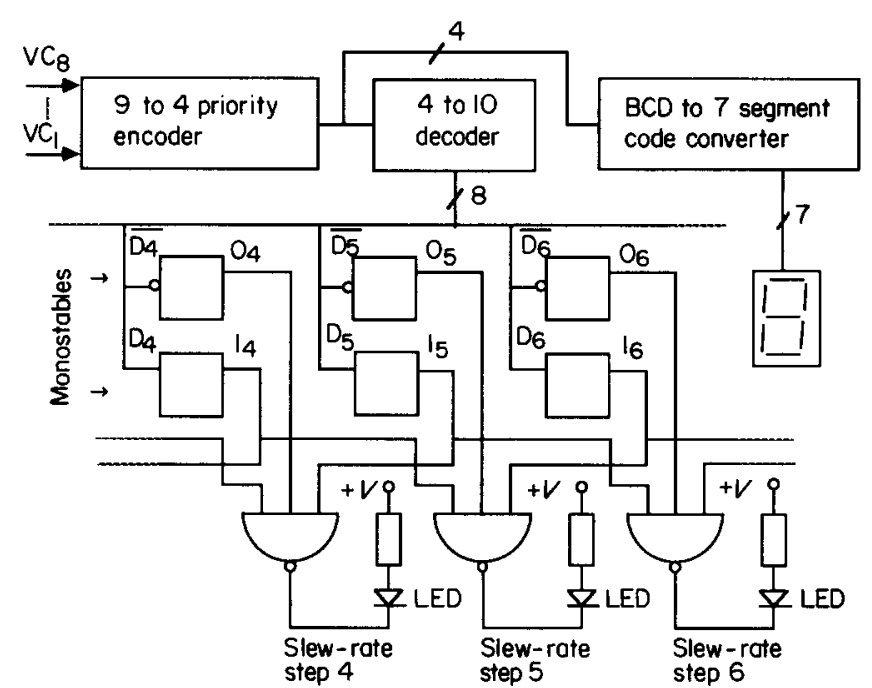

Figure 7 Logic circuit to display number of activated step and to signal that flux error of the SOUID being simulated is larger than $\Phi_{0} / 4$. This is indicated by the slew rate LEDs. $V C_{1} \ldots V C_{8}$ are outputs of voltage generators of Figure $5 a$

modulation signal is set to $\pm 0.25 \Phi_{\mathrm{o}}$. A sinusoidal input signal is applied and its slew rate is increased slowly (this can be done by fixing the frequency and increasing the amplitude). Correspondingly, the error signal $V_{\mathrm{e}}(s)$ in Figure $2 a$ will increase and $E_{1}$ will go beyond the set range, leading to a switch of $E_{2}$. This unlocking of the system is detected by the seven segment display.

There is, however, one complication. Being near to but still below the maximum value of the error signal, spikes from the environment may lead to $E_{1}$ momentarily exceeding the set range, directly followed by a return to normal values. The voltage comparator is fast enough to detect this and thus it is signalled on the display. This short disturbance does not change $E_{2}$ so the system is still locked. Therefore, a change in the seven segment display is not proof of a maximum slew rate occurrence.

An additional circuit was designed that discriminates between the activation of the voltage comparators due to spikes and due to the exceeding of the slew rate limit. In the first case the activation sequence of the voltage comparators is $\mathrm{VC}_{i}, \mathrm{VC}_{i+1}, \mathrm{VC}_{i}$, and in the second case $\mathrm{VC}_{i}, \mathrm{VC}_{i+1}, \mathrm{VC}_{i}, \mathrm{VC}_{i-1}, \mathrm{VC}_{i}$. The criterion for the slew rate limit is that the latter sequence occurs within $0.1 \mathrm{~ms}$. This time interval is obtained with monostables and the occurrence is detected by a LED.

Part of Figure 7 depicts the circuit. The number of the step codified in binary is decoded with a four to ten decoder and this is used to activate the monostables, $D_{i}$. Each line drives two monostables; one is sensitive to the rising edge and the other to the falling edge. The monostables connected to the output line $i$ detect the incoming level, $\mathrm{I}_{i}$, into step $i$ and the outgoing levels $\mathrm{O}_{i}$, from step $i$, respectively. To perform the desired function the LED is activated if, within the time interval of the monostables, sequences of the form $\mathrm{O}_{i}{ }^{*} \mathrm{I}_{i+1}{ }^{*} \mathrm{I}_{i-1}$ or $\mathrm{O}_{i}{ }^{*} \mathrm{I}_{i-1}{ }^{*} \mathbf{I}_{i+1}$ are detected.

\section{Tests on the circuit}

The following tests have been performed on the simulator, while obtaining input currents with voltage signals 
through high value resistors:

1 Output characteristics of the simulator and output from the generator of voltage references obtained with a low frequency input signal of $100 \mathrm{~Hz}$.

2 Operation of the simulator in a configuration equivalent to that of SQUID electronics. A square wave modulation of $100 \mathrm{kHz}$ and $\pm 0.25 \Phi_{\mathrm{o}}$ amplitude is used.

3 Dynamic behaviour for small sinusoidal input signals $\left(\leqslant 0.25 \Phi_{0}\right)$.

4 Noise measurements.

5 Slew rate experiments.

Each of these tests will now be considered in more detail

For the first test, the system is internally adjusted with $i_{\mathrm{p}}$ set to start at step number 4 . Then a low frequency $(100 \mathrm{~Hz})$ triangular wave of current equivalent to $\pm 4.5 \Phi_{\mathrm{o}}$ and $100 \mathrm{~Hz}$ is applied as the input. Figure 8 represents a photograph of the input signal (triangular wave), the output from the generator of voltage references (stair-step shapes) and the nine output steps (V shapes). This last signal has been taken from the input of the attenuator to give a better display.

The second test was performed using the scheme shown in Figure 9a. The input signal is a triangular wave of $100 \mathrm{~Hz}$ and amplitude $4 \Phi_{\mathrm{o}}$. Additionally, a square wave of $100 \mathrm{kHz}$ and $0.25 \Phi_{0}$ amplitude has been applied as a modulation signal. An amplifier with $\mathrm{Av}=890$ and a multiplier with a scale factor of $1 / 10$ are used to detect the response of the simulator. The reference input of the multiplier is a sinusoidal wave of $\pm 10 \mathrm{~V}$ and $f=100 \mathrm{kHz}$, adjusted in phase. Using this arrangement the results in Figure $9 b$ have been obtained, these being the simulated results for a SQUID in operation. The forward transfer ratio, $S$, can be calculated from Figure $9 b$ as $100.6 \mu \mathrm{V} / \Phi_{0}$, which is close to the expected value.

To measure the small signal response of the simulator for test 3, the scheme shown in Figure $9 a$ was used and the triangular wave was replaced by a sinusoidal signal with an amplitude smaller than $0.25 \Phi_{0}$. Now the system

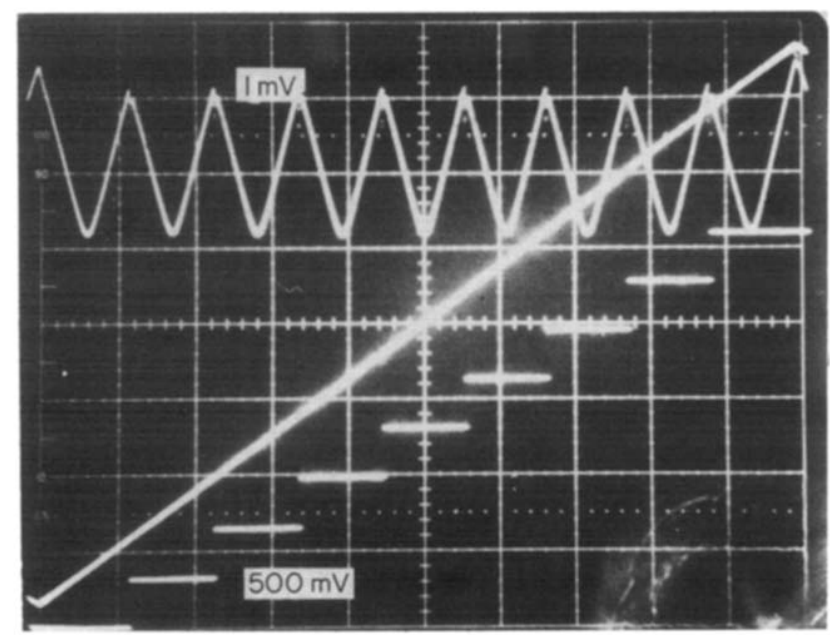

Figure 8 Nine steps of simulator (V shapes) and output from generator of voltage references obtained with a triangular input signal of $100 \mathrm{~Hz}$. Scale for $V$ shapes: horizontal $0.5 \mu$ s division $^{-1}$. vertical $1 \mathrm{mV}$ division $^{-1}$. Scale for voltage references: $500 \mathrm{mV}$ division $^{-1}$. Input is uncalibrated to fit into the screen. $V$ shapes have been taken from the input of attenuator for better display
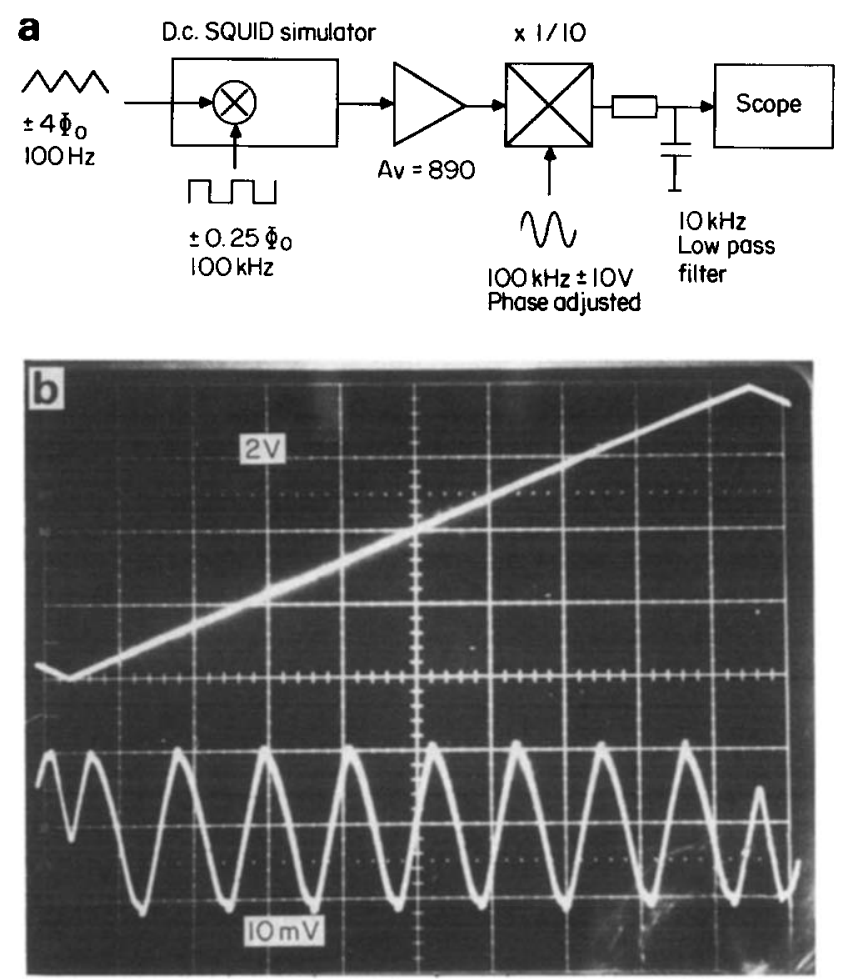

Figure 9 (a) Arrangement used to obtain Figure $9 b$. Input signal equivalent to $\pm \mathbf{4} \Phi_{\mathrm{o}}$ is modulated with a square wave of $\pm \mathbf{0 . 2 5} \Phi_{\mathrm{o}}$ and a frequency of $100 \mathrm{kHz}$. (b) Nine steps of simulator obtained after detection. Scale for triangular wave with long period (input signal): horizontal uncalibrated $\leqslant 0.5 \mu$ division $^{-1}$, vertical $2 \vee$ division $^{-1}$. Scale for output: $10 \mathrm{mV}^{\text {division }}{ }^{-1}$

operates in one step and the frequency response can be measured. At the output of the preamplifier not only is the basic signal with frequency $f$ measured but also harmonics with frequencies $100 \mathrm{kHz} \pm f, 200 \mathrm{kHz} \pm f \ldots$, due to the modulation frequency of $100 \mathrm{kHz}$. To observe the output properly a low pass filter of $20 \mathrm{kHz}$ and $24 \mathrm{~dB} /$ octave roll-off was applied, this being adequate to test the simulator in the frequency range up to $500 \mathrm{~Hz}$. This range covers all biomagnetic experiments planned with the multichannel d.c. SQUID system. The measured frequency behaviour was completely determined by the $20 \mathrm{kHz}$ filter. Thus it is concluded that the simulator has a flat frequency response in the range of biomagnetic experiments (up to $500 \mathrm{~Hz}$ ).

For the fourth test, an upper limit for the noise contribution of the simulator was indirectly evaluated. A monolithic preamplifier was used (gain $=1000$ ) with equivalent noise parameters at the input at $100 \mathrm{kHz}$ of $E_{\mathrm{n}}=1.8 \mathrm{nV} / \mathrm{Hz}^{1 / 2}$ and $I_{\mathrm{n}}=1 \mathrm{pA} / \mathrm{Hz}^{1 / 2}$. No increase in the noise level was observed after connection of the simulator to the preamplifier. Due to the low output resistance of the simulator, the current noise contribution is negligible. Therefore the voltage noise of the simulator is below $1.8 \mathrm{nV} / \mathrm{Hz}^{1 / 2}$.

A second test was made using the simulator and the preamplifier with d.c. SQUID electronics in feedback mode operation. The transfer ratio of the system is $1 \mathrm{~V} / \Phi_{\text {o }}$ and with the forward transfer ratio of the simulator at $100 \mu \mathrm{V} / \Phi_{\mathrm{o}}$ it follows that the ratio between the SQUID electronics output and preamplifier input is $10^{4}$. Using this configuration a noise level of $18 \mu \mathrm{V} / \mathrm{Hz}^{1 / 2}$ was measured at the output, and it was concluded that the preamplifier is the most noisy part of the circuit. 


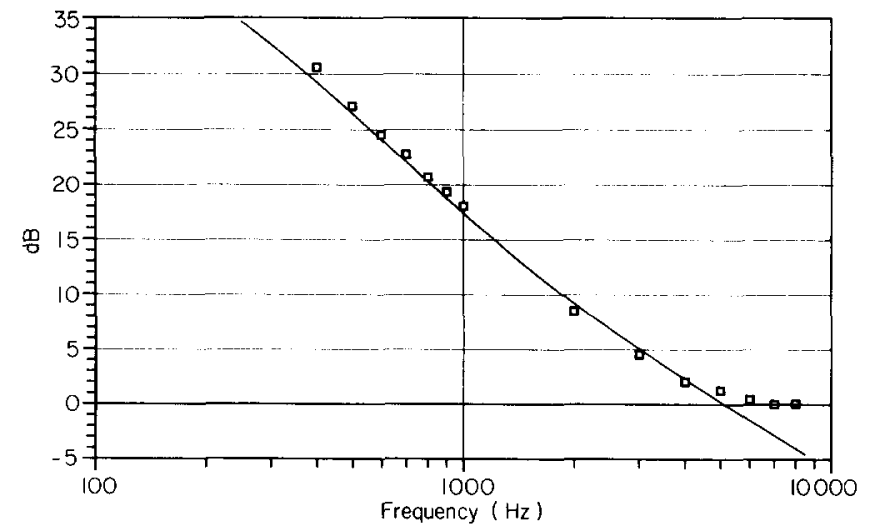

Figure 10 Reconstruction of open loop transfer function of system through slew rate measurements achieved with simulator. Values calculated from $\omega_{c}, \omega_{z}$ and $\omega_{p} ; \square$, values obtained from slew rate measurements (the differences are of the order of $0.7 \mathrm{~dB}$ )

For the final test, the slew rate limit of a SQUID system is given by the maximum rate of change of input flux, $\mathrm{d} \Phi_{\mathrm{i}} / \mathrm{d} t$, that can be followed without interrupting the closed loop operation. This maximum depends on the open loop transfer function of the SQUID electronics ${ }_{3}$. For a system with one dominant pole it holds that $\mathrm{d} \Phi_{\mathrm{i}} / \mathrm{d} t_{\max }=\omega_{\mathrm{c}} \times \Phi_{\mathrm{o}} / 4, \omega_{\mathrm{c}}$ being the (angular) frequency at the point where the open loop gain is $0 \mathrm{~dB}$. For a system with two poles and one zero the maximum is given by $\mathrm{d} \Phi_{\mathrm{i}} / \mathrm{d} t_{\max }=\left(\omega_{\mathrm{c}} \times \omega_{\mathrm{z}} / \omega\right) \Phi_{\mathrm{o}} / 4$ for values $\omega<\omega_{\mathrm{z}}$, and $\mathrm{d} \Phi_{\mathrm{i}} / \mathrm{d} t_{\max }=\omega_{\mathrm{c}} \times \Phi_{\mathrm{o}} / 4$ for $\omega>\omega_{\mathrm{z}}$, where $\omega_{\mathrm{z}}$ is the frequency of the zero signal and $\omega$ the frequency of the input signal

The slew rate experiments were performed with d.c. SQUID control electronics of the second type. For this system, $\omega_{\mathrm{c}}=2 \pi \times 15600$, the pole frequency, $\omega_{\mathrm{p}}=2 \pi \times$ 400 and $\omega_{\mathrm{z}}=2 \pi \times 1250$. The open loop gain, 20 $\log |G(j \omega) \times H(j \omega)|$, of this system is calculated with these values and is depicted as a continuous line in Figure 10. $G(j \omega)$ and $H(j \omega)$ are the frequency dependent transfer functions of the forward and the feedback path of the loop. The aim of the experiment is to reconstruct the open loop transfer function with the information obtained from the slew rate measurements. It has been shown ${ }^{3}$ that $\mathrm{d} \Phi_{\mathrm{i}} / \mathrm{d} t_{\max }=\Phi_{\mathrm{i}} \times \omega$ and $\omega_{\mathrm{c}} \times \omega_{\mathrm{z}} / \omega^{2}=$
$|G(j \omega) \times H(j \omega)|$ for values $\omega<\omega_{z}$, and $\omega_{\mathrm{c}} / \omega=$ $|G(j \omega) \times H(j \omega)|$ for $\omega>\omega_{\mathrm{z}}$. So, it follows that $20 \log |G(j \omega) \times H(j \omega)|=20 \log \left(4 \times \Phi_{\mathrm{i}} / \Phi_{\mathrm{o}}\right)$ for both cases. This is the formula used to obtain the open squares in Figure 10. Prior to the slew rate measurements, the simulator and the d.c. SQUID electronics have been connected in a closed loop and the open loop gain has been adjusted to obtain the desired closed loop transfer function.

For the frequency range $\omega_{\mathrm{p}}<\omega<\omega_{\mathrm{c}}$ the measured values are within $0.7 \mathrm{~dB}$ of the theoretical ones. This gives a level of error of $\approx 8 \%$ for the experimental measurement of slew rate using this procedure.

\section{Concluding remarks}

An electronic circuit was developed that simulates nine steps of a SQUID. This simple instrument is extremely suited to testing and adjustment of multichannel d.c. SQUID electronics. The work can be performed in an electronics laboratory, as there is no need for cryogenic infrastructure.

The main parameters of a d.c. SQUID, such as forward transfer ratio, feedback coupling and dynamic resistance could be easily implemented and modifications of these values can be simply realized. Thus the system can be adapted to suit each specific SQUID development.

\section{Acknowledgements}

A. Martinez is supported in part by the Diputación General de Aragón, Zaragoza, Spain.

\section{References}

1 Henry, R.W. and Prober, D.E. Rev Sci Instrum (1981) 52902

2 Tuckerman, D.B. Rev Sci Instrum (1978) 49835

3 Rillo, C., Veldhuis, D. and Flokstra, J. IEEE Trans Instrum Meas (1987) IM-36 770

4 Toumazou, C. and Lidgey, F.J. IEE Proc G (1987) 1347

5 Toumazou, C. and Lidgey, F.J. Electronics and Wireless World (1987) 931115

6 Wilson, B. Wireless World (1981) 8747

7 Lidgey, F.J. Wireless World (1979) 8557 